\title{
A NOTE ON THE INVERTIBLE IDEAL THEOREM
}

\author{
by ANDY J. GRAY
}

(Received 20 June, 1982)

1. Introduction. This note is devoted to giving a conceptually simple proof of the Invertible Ideal Theorem [1, Theorem 4.6], namely that a prime ideal of a right Noetherian ring $R$ minimal over an invertible ideal has rank at most one. In the commutative case this result may be easily deduced from the Principal Ideal Theorem by localizing and observing that an invertible ideal of a local ring is principal. Our proof is partially analogous in that it utilizes the Rees ring (defined below) in order to reduce the theorem to the case of a prime ideal minimal over an ideal generated by a single central element, which can be easily dealt with by adapting the commutative argument in [8]. The reader is also referred to the papers of Jategaonkar on the subject $[5,6,7]$, particularly the last where another proof of the theorem appears which yields some additional information.

I would like to extend my thanks to my supervisor, Dr C. R. Hajarnavis, for his help and encouragement throughout.

2. The proof. We begin with some essential definitions and notation. Let $R$ be any ring, $X$ an ideal of $R$. Then $R[X t]$ denotes the subring

$$
\left\{x_{j} t^{j}+\ldots+x_{0} \mid x_{i} \in X^{i}, i \leqslant j, j \in \mathbb{N}\right\}
$$

of the polynomial ring over $R$ in a commuting indeterminate $t$. We shall more loosely write

$$
R[X t]=R \oplus X t \oplus X^{2} t^{2} \oplus \ldots
$$

Further, the Rees ring of $R$ at $X$, denoted $R\left[t^{-1}, X t\right]$, is given by

$$
\begin{aligned}
R\left[t^{-1}, X t\right] & =R[X t]\left[t^{-1}\right] \\
& =\ldots \oplus R t^{-2} \oplus R t^{-1} \oplus R \oplus X t \oplus X^{2} t^{2} \oplus \ldots
\end{aligned}
$$

The following proposition, and indeed its proof, are the appropriate variants on the Hilbert Basis Theorem. For $f \in R[X t]$, we let $\operatorname{deg}(f)$ and $h c(f)$ denote the degree and highest coefficient of $f$ in the obvious sense.

Proposition 2.1. Let $R$ be a right Noetherian ring, $X$ an invertible ideal of $R$. Then the rings $R[X t]$ and $R\left[t^{-1}, X t\right]$ are right Noetherian.

Proof. As $R\left[t^{-1}, X t\right]$ is a homomorphic image of a polynomial extension of $R[X t]$ in a commuting indeterminate, it clearly suffices to show that $R[X t]$ is right Noetherian.

Let $A$ be a right ideal of $R[X t]$; we shall show that $A$ is finitely generated. Define

$$
\begin{aligned}
B_{n}^{\prime}=\{\operatorname{hc}(f) \mid \operatorname{deg}(f) & =n \text { or } f=0, \text { with } f \in A\}, \\
B_{n} & =B_{n}^{\prime} X^{-n} .
\end{aligned}
$$

Glasgow Math. J. 25 (1984) 27-30. 
Then $B_{n}^{\prime}$ is a right ideal of $R$ contained in $X^{n}$, so that

$$
B_{0} \subset B_{1} \subset B_{2} \subset \ldots
$$

is a chain of right ideals of $R$. Now there is some integer $k$ with $B_{k}=B_{k+i}$ for all $i \geqslant 0$, or equivalently $B_{k}^{\prime} X^{i}=B_{k+i}^{\prime}$. For $i=1, \ldots, k$, choose generators $b_{i, 1}, \ldots, b_{i, m_{i}}$ for $B_{i}^{\prime}$ over $R$, and $f_{i, j} \in A$ with $\operatorname{deg}\left(f_{i, j}\right)=i$ and $h c\left(f_{i, j}\right)=b_{i, j}$. We claim that the $f_{i, j}$ together generate $A$. Let $g \in A, h c(g)=b$ and $\operatorname{deg}(g)=v$. Suppose first that $v>k$. Then $b \in B_{v}^{\prime}=B_{k}^{\prime} X^{v-k}$ and so $b=\sum_{i} b_{k, j} x_{j}$ with $x_{j} \in X^{v-k}$. Thus $x_{j} t^{v-k} \in R[X t]$; hence

$$
g-\sum_{j} f_{k, j} x_{j} t^{v-k}
$$

lies in $A$ and has degree strictly less than that of $g$. We may thus reduce to the case where $v \leqslant k$. Repeating a somewhat simpler version of the above argument, we finally obtain

$$
g \in \sum_{i, j} f_{i, j} R[X t]
$$

as required.

The above proposition has two interesting consequences. Firstly, the usual commutative argument shows that the ideal $X$ has the strong right Artin-Rees property: for each right ideal $E$ of $R$ there exists an integer $n$ such that for all $s>n$,

$$
E \cap X^{s}=\left(E \cap X^{n}\right) X^{s-n} \text {. }
$$

Secondly, it is easily checked that there is an isomorphism between the factor ring $R\left[t^{-1}, X t\right] / t^{-1} R\left[t^{-1}, X t\right]$ and the graded ring of $R$ at $X$ (the invertibility of $X$ is irrelevant here). It is well known that the right Noetherian condition in the graded ring implies the same condition in the completion, so that the completion of a right Noetherian ring at an invertible ideal is again right Noetherian. In particular, it is immediate that the $X$-adic completion of a Dedekind prime ring at any non-zero ideal $X$ is Noetherian. (Such a completion is in fact a finite direct sum of prime principal right and left ideal rings; see [4] for the case where $X$ is a maximal ideal.) Indeed, as the Jacobson radical of a hereditary Noetherian prime ring is invertible when it is non-zero [3, Theorem 4.13], part of the main result of [2] has also been proved.

Our aim is now to indicate a reduction of the proof of the Invertible Ideal Theorem to the following case, which may be proved by a straightforward modification of [8, Theorem 142], replacing a length argument by a reduced rank one.

THEOREM 2.2. Let $R$ be a right Noetherian ring, $a \in R$ a central element and $P$ a prime ideal of $R$ minimal over $a R$. Then $\operatorname{rank}(P) \leqslant 1$.

Now let $X$ be an ideal of a ring $R$ and $K$ a right ideal of $R$. Then $K[X t]$ denotes the right ideal 
of $R[X t]$. If $X$ is invertible, then $K^{*}$ denotes the right ideal

$$
\ldots \oplus\left(K X^{-2} \cap R\right) t^{-2} \oplus\left(K X^{-1} \cap R\right) t^{-1} \oplus K \oplus K X t \oplus K X^{2} t^{2} \oplus \ldots
$$

of $R^{*}=R\left[t^{-1}, X t\right]$. The following two lemmas form the technical basis for our argument.

LeмmA 2.3. Let $R$ be a ring with an invertible ideal $X$.

(a) If $P$ is a prime ideal of $R$ such that $P X=X P$, then $P[X t]$ and $P^{*}$ are prime ideals of the rings $R[X t]$ and $R^{*}$ respectively.

(b) Suppose that $R$ is right Noetherian. Let $N / X$ be the nilpotent radical of $R / X$, and $P_{1}, \ldots, P_{n}$ the primes of $R$ minimal over $X$. If $X$ commutes with each $P_{i}$ (i.e. $P_{i} X=X P_{i}$ ), then $P_{1}^{*}, \ldots, P_{n}^{*}$ are the primes of $R^{*}$ minimal over $X^{*}$ and the nilpotent radical of $R^{*} / X^{*}$ is $N^{*} / X^{*}$.

Proof. (a) As $P X=X P, P[X t]$ and $P^{*}$ are ideals of the appropriate rings. Let $f(t)=a_{0}+\ldots+a_{n} t^{n}$ and $g(t)=b_{0}+\ldots+b_{s} t^{s}$ be elements of $R[X t]$ with $g(t) R[X t] f(t) \subset$ $P[X t]$ and $f(t) \notin P[X t]$. We claim that $g(t) \in P[X t]$. We may assume that

$$
f(t)=a_{m} t^{m}+\ldots+a_{n} t^{n}
$$

where $a_{m} \notin P X^{m}$ and $a_{i}=0$ for $i<m$. Now $b_{0} R a_{m} \subset P X^{m}$ so that $b_{0} R a_{m} X^{-m} \subset P$. As $a_{m} X^{-m} \not \subset P$, one has $b_{0} \in P$. Therefore

$$
\left(g(t)-b_{0}\right) R[X t] f(t) \subset P[X t]
$$

so that

$$
\begin{aligned}
X^{-1} t^{-1}\left(g(t)-b_{0}\right) R[X t] f(t) & \subset X^{-1} t^{-1}\left(P[X t] \cap\left(X t \oplus X^{2} t^{2} \oplus \ldots\right)\right) \\
& =X^{-1} t^{-1}\left(P X t \oplus P X^{2} t^{2} \oplus \ldots\right) \\
& =P[X t] .
\end{aligned}
$$

Each polynomial in the set $X^{-1} t^{-1}\left(g(t)-b_{0}\right)$ has degree strictly less than that of $g$, and it follows by induction that $g \in P[X t]$, so that $P[X t]$ is prime. That $P^{*}$ is a prime ideal of $R^{*}$ follows easily from the observation that for each $a \in R^{*}$ there is some $r \geqslant 0$ with $X^{r} t^{r} a \subset R[X t]$.

(b) By part (a), the $P_{i}^{*}$ are prime ideals of $R^{*}$. As $N^{*}$ is their irredundant intersection, it is sufficient to check that $N^{*}$ is indeed nilpotent modulo $X^{*}$.

Lemma 2.4. Suppose that $X$ is an invertible ideal of a ring $R$. Then $X^{*}$ is generated by a central element of $R^{*}$.

Proof. It is easily seen that $X^{*}=t^{-1} R^{*}$.

Assembling these results, we can now prove the Invertible Ideal Theorem.

Theorem 2.5. [1, Theorem. 4.6]. Let $R$ be a right Noetherian ring, $X$ an invertible ideal of $R$ and $P$ a prime ideal minimal over $X$. Then $\operatorname{rank}(P) \leqslant 1$.

Proof. Suppose that rank $(P)>1$, so that there is a proper chain of primes $P \supset Q \supset Q^{\prime}$ of $R$. Let $N / X$ be the nilpotent radical of $R / X$. Then the ideals $X^{-1} N X$ and $X N X^{-1}$ are 
again nilpotent modulo $X$, from which it follows that $N=X^{-1} N X$. If $P_{1}, \ldots, P_{n}$ are the primes of $R$ minimal over $X$, then each $X^{-1} P_{i} X$ is again a prime of $R$ and $X^{-1} P_{1} X, \ldots, X^{-1} P_{n} X$ is seen to be the complete set of primes of $R$ minimal over $X$ by applying [1, Lemma 4.1]. Hence conjugation by $X$ permutes the $P_{i}$, so that after replacing $X$ by a suitable power we may assume that $X$ commutes with the $P_{i}$, and in particular with $P$. As $X$ lies in neither $Q$ nor $Q^{\prime}$, Lemma 4.2 of [1] shows that $X$ also commutes with these primes. Therefore by Lemma 2.3(a), $P^{*} \supset Q^{*} \supset Q^{\prime *}$ is a proper chain of primes of $R^{*}$, and $P^{*}$ is minimal over $X^{*}$ (Lemma 2.3(b)). Combining Lemma 2.4 and Theorem 2.2 yields the desired conclusion.

\section{REFERENCES}

1. A. W. Chatters, A. W. Goldie, C. R. Hajarnavis and T. H. Lenagan, Reduced rank in Noetherian rings, J. Algebra 61 (1979), 582-589.

2. V. K. Deshpande, Completions of Noetherian hereditary prime rings, Pacific I. Math. 90 (1980), 285-297. $86-104$.

3. D. Eisenbud and J. C. Robson, Hereditary Noetherian prime rings, J. Algebra 16 (1970),

4. W. D. Gwynne and J. C. Robson, Completions of non-commutative Dedekind prime rings, J. London Math. Soc (2) 4 (1971), 346-352.

5. A. V. Jategaonkar, Relative Krull dimension and prime ideals in right Noetherian rings, Comm. Algebra 4 (1974), 429-468.

6. A. V. Jategaonkar, Principal Ideal Theorem for Noetherian P.I. rings, J. Algebra 35 (1975), $17-22$.

7. A. V. Jategaonkar, Relative Krull dimension and prime ideals in right Noetherian rings: An addendum, Comm. Algebra 10 (1982), 361-366.

8. I. Kaplansky, Commutative rings (Allyn and Bacon, 1970).

Mathematics InSTITUTE

UNIVERSITY OF WARWICK

COVENTRY CV4 7AL 